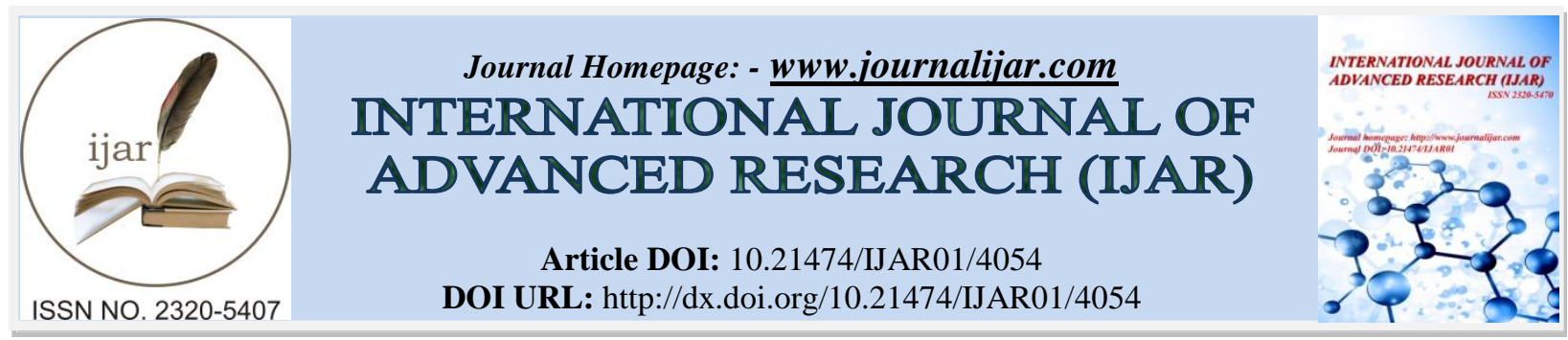

RESEARCH ARTICLE

\title{
NOVEL ENERGY-SAVING TRANSMISSIONS IN OFDMA-BASED MULTICARRIER BASE STATIONS BY RADIO RESOURCE MANAGEMENT.
}

Y. Hareen Tej, Nikhil Raidu and Ravi Kumar C. V.

School of electronics and communication engineering, Vellore Institute of Technology, vellore, Tamil nadu, India.

\section{Manuscript Info}

Manuscript History

Received: 26 February 2017

Final Accepted: 27 March 2017

Published: April 2017

Key words:-

Component carrier, Energy saving,

Green communication technology,

OFDMA, SUI channel model

\begin{abstract}
As mobile knowledge traffic levels have enhanced exponentially, leading to rising energy prices in recent years, the demand for and development of inexperienced communication technologies has resulted in varied energy-saving styles for cellular systems. At an equivalent time, recent technological advances have allowed multiple element carriers (CCs) to be at the same time utilized in a very base station (BS), a development that has created the energy consumption of BSs a matter of accelerating concern. Digital signal process came ways from wire-based glass fiber communication to wireless-based high rate supported communication models. Radio waves supported long distance satellites, radars to microwave supported mobiles has modified in terms of technology and rate in last 20 years. As mobile data traffic increases there is unavailability of power at base station. So we are going to implement adaptive resource scheduling algorithm to provide power adaptively.
\end{abstract}

Copy Right, IJAR, 2017,. All rights reserved.

\section{Introduction:-}

Nowadays numbers of users are increased in Tele-communication industry and they require high data rate, and network access from anywhere. So the wireless communication technology needs more energy or power, while using more power it releases some unwanted gases called $\mathrm{CO} 2$ into the environment, the International Telecommunication unit(ITU) announced that the ICT (information and communication Technology) releases 2\% $2.5 \%$ of total greenhouse gas emission. As a result of that, some amount of energy is wasting. In order to overcome this problem we are going for Rate and Power Control method, by using this we can use energy in efficient manner. And this method plays a major role in future Wireless telecommunication technology. In present scenario we are using 3GPP and Long Term Evaluation in cellular communication these technologies provides high speed data, significant spectral efficiency etc. we can use energy in efficient manner by using these technologies called

1. MIMO

2. OFDMA

3. $\mathrm{RB}$

4. Sub channel assignment.

In the above process each user may allocate to the RB (Resource Block) by resource allocation algorithm, the RB provides good QOS (Quality of Service) and efficient use of energy. Energy efficient algorithm for allocating sub carrier to the user shows water filling packet scheduling algorithm and shows that Resource Blocks is allocated to the users by resource scheduling.

Corresponding Author:- Yhareen Tej.

Address:- School of electronics and communication engineering, Vellore Institute of Technology, vellore 
The resource allocation problem needs M2M and $\mathrm{H} 2 \mathrm{H}$ user's energy efficient resource allocation in uplink LTE networks under statistical QoS provisioning the dual problem. This paper gives Downlink transmission and supports the following

1. Real Time and

2. Non Real Time

The Rate and Power Control method may give more preference to real time users because they use the technology continuously, where as non real time users use the technology in some specific times. So this method gives more power when number of user's increases. And this is the efficient method to use power in a efficient manner in present scenario.

\section{Background:-}

(A) Radio Resource Allocation:-

In radio resource allocation previously, we are used TDMA, FDMA techniques. In TDMA time is divided into frames then frames are divided into subframes.10ms frames are divided as ten $1 \mathrm{~ms}$ sub-frames. Then these sub frames divided into time slots. Each subframe is divided into two slots each slot will contain $0.5 \mathrm{~ms}$ and every slot will contain 7 OFDM symbols with normal cyclic prefix. In FDMA each channel is divided into sub channels as in frequency. One sub channel will contain $180 \mathrm{kHz}$ and every sub channel will contain 12 consecutive and equally spaced OFDM sub-carriers. Resource block (RB) will obtain by performing intersection between the sub channels of frequency domain and time domain.

\section{(B) Green Wireless Communication:-}

Green wireless communication technology is working on efficient energy utilization of future communication networks.

There are four main research topics in this domain they are:

1. Network architectures.

2. Radio resource management (adaptive)

3. Interference management.

4. Energy consumption models metrics.

\section{(C) OFDMA:-}

Orthogonal frequency division multiple access (OFDMA) is a multi-access version of the orthogonal frequency division multiplexing (OFDM). The main difference between OFDM and OFDMA is in OFDM only single user will use the data, but in OFDMA multiple users will use the data. In OFDMA total bandwidth is divided into sub channels with sub-carriers and each sub-carrier are modulated with a lower data rate. At a time these lower data rate streams will transmit through the sub-carriers, then we can achieve the high speed of data transmission OFDMA will use the advantages of OFDM technique, i.e. Multipath mitigation and interference cancelation and combat against channel fading effect, in OFDMA there will a large number of sub-carriers. Those sub-carriers should transform carefully in a scheduled manner. According to the channel conditions or user's demands transmission can be done so dynamic frequency allocation is achieved in OFDMA.

\section{Proposed Method:-}

\section{(A) Admission Control Mechanism:-}

In this implementation we are going to consider a very high number of users. As users are suddenly increasing and decreasing, the fixed resource allocation algorithm will provide the data in continuous fashion, so there is very high data loss. That data loss is nothing but power and energy loss is very high.

As session incoming to the system we are separating them as real time users and nonreal time users. RT users are using data continuously and very high data requirement is there but for nonreal time users less data requirement is there. As shown in Fig.1 


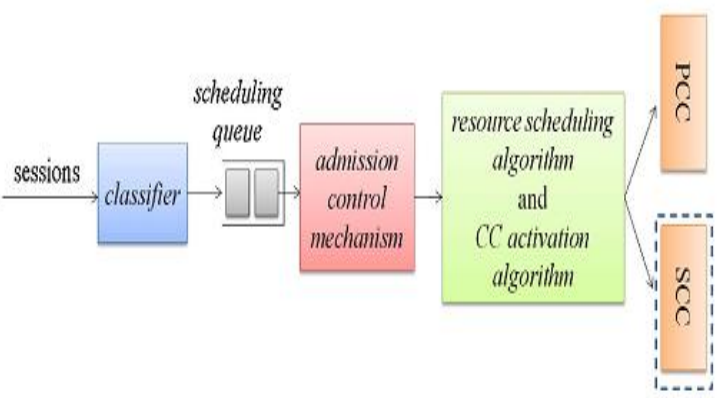

Fig 1:- Admission Control Mechanism

\section{(B) Affirmation Control Mechanism:-}

By using this mechanism we are going to check the requirements of the data rate. We know the basic formula of capacity of channel. Below one data rate formula is there which is derived from it. Based on $\boldsymbol{r}_{\boldsymbol{m}, \boldsymbol{j} \boldsymbol{n}}^{(\boldsymbol{k})}$ can be given as

$$
r_{m, j \_n}^{(k)}=\beta \log _{2}\left(1+\frac{K P_{m, j}^{(k)}\left|H_{j_{-} n}^{(k)}\right|}{\beta N_{0}}\right)
$$

Above the sub channel noise $N_{0}$ is the commotion power unearthly thickness, $\mathrm{j}$ and client session $\mathrm{n}$ on $\mathrm{CC} \mathrm{k}$, and bandwidth available $\beta=12 \cdot 15000$ is the data transmission in $\mathrm{Hz}$ for a RB, one RB is having 12 subcarriers what's more, each subcarrier is characterized to have $15000 \mathrm{~Hz}, \mathrm{~K}=-1.5 \log (5 \mathrm{~B} \mathrm{E} \mathrm{R})$, where BER is the wanted (steady) piece blunder rate, and $\boldsymbol{P}_{\boldsymbol{m}, \boldsymbol{j}}^{(\boldsymbol{k})}$ is the required transmission energy to accomplish $\boldsymbol{r}_{\boldsymbol{m}, \boldsymbol{j}_{\mathbf{n}}}^{(\boldsymbol{k})}$ under the plan structure in (1). In light of (1), the transmission force of $(m, j)_{\boldsymbol{R} \boldsymbol{B}}$ on $\mathrm{CC} \mathrm{k}$ can be given as

$$
P_{m, j}^{(k)}=\frac{\beta N_{0}}{K\left|H_{j \_n}^{(k)}\right|}\left(2^{\frac{r_{m, j \_n}^{(k)}}{j}}-1\right)
$$

From the above formula for power we can easily calculate the energy requirement in CCk carrier, $E_{k}$ can be calculated as

$$
E_{k}=\frac{t_{\text {Sub_frame }}}{2} \sum_{(m, j)_{\boldsymbol{R B}} \in \Omega_{k}} \boldsymbol{P}_{\boldsymbol{m}, \boldsymbol{j}}^{(\boldsymbol{k})}
$$

Where $t_{\text {Sub_frame }}$ length of each subframe, $\Omega_{k}$ is the arrangement of all RBs in each subframe of CC k. 


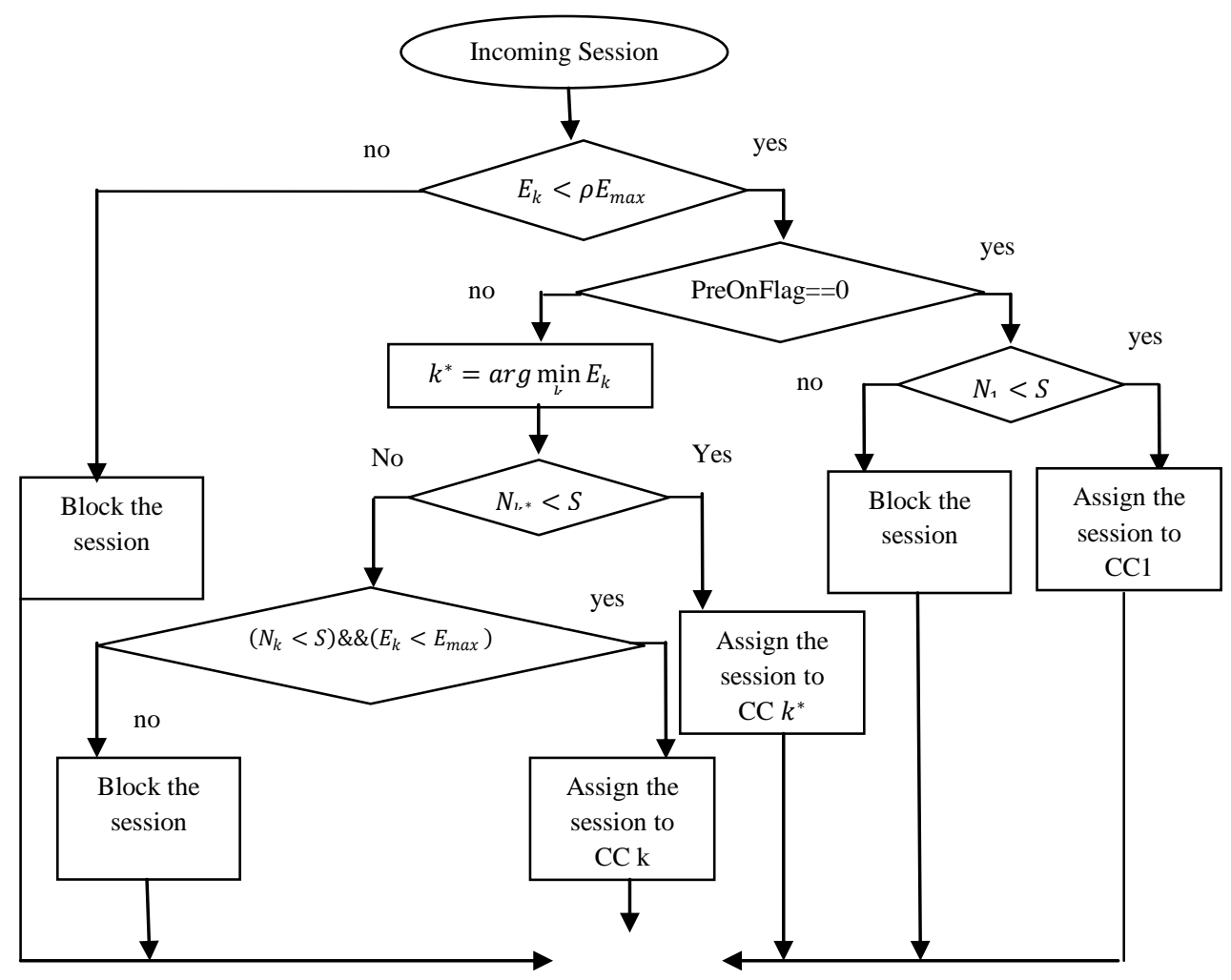

Figure 2:- Flow chart of the admission control mechanism

Below control mechanism algorithm is there which shows that if power requirement is very high at that time how we can access to the SCC. We are using 'preonflag' to know the status of the SCC. That is either SCC is available or not.

If In PCC available power is utilized then we have to go for SCC to access more users. Those who require again less energy is checked by using $\arg \min E_{k}$ condition. If $\mathrm{N} 1<\mathrm{S}$, where $N_{k}$ speaks to the number of client sessions in the framework on CC k, then we can use again PCC.Instead of SCC, we can use PCC.

In the other case, if PreOnFlag==1, $\mathrm{CC} \mathrm{k}^{*}$ that has the base Ek will be picked. Taking after that, the instrument will check whether $\mathrm{N}_{-}\left(\mathrm{k}^{*}\right)<\mathrm{S}$. In case yes, $\mathrm{CC} \mathrm{k}^{*}$ will be rolled out to the new session; something else, the instrument will advance check whether N_k $<\mathrm{S}$ besides, E_k $<$ E_max to make sense of whether the new session can get to CC $\mathrm{k}$. See that the operation and check of the framework is executed toward the begin of each subframe.

\section{(C) Objective of the Novel Energy-Saving Transmission:-}

Scheme in sight of the thought of framework demonstrate, the combination vitality utilization in every subframe at the SB handsets is pointed to be decreased, whereas maintaining the block probability of all shopper sessions, the bottom needed data rates for each reasonably purchasers, and also the reasonableness among all purchasers in associate degree satisfactory level. To profitably and adequately accomplish the higher than objective, a completely unique vitality economical arrange, which includes associate degree quality booking calculation in Section III and a $\mathrm{CC}$ initiation calculation in Section IV, is projected

\section{(D) Resource Scheduling Algorithm:-}

The introduced plus booking calculation incorporates 2 calculations that area unit severally planned for the operation as takes after:

1) Vitality versatile rate management calculation (EARCA) additionally,

2) Radioresource allocation algorithm (RRAA). The RRAA calculation is more isolated into 2 sub algorithms named

B.1) knowledge transfer capability task calculation (BAA) and 
B.2) plus piece designation calculation (RBAA), separately.

EARCA is meant to powerfully alter the NRT client's assigned limit visible of his/her means misfortune criticism and also the current utilized vitality. When the NRT client's data rate is about, BAA decides what range of RBs need to be parceled out to every shopper session, whereas RBAA is used to encourage decide the set of RBs for those sessions.

\section{(E) Radio Resource Allocation Algorithm (RRAA):-}

RRAA is made public on the premise of the plus allotment approach used, for its process many-sided nature advantage. Pseudo codes for purpose by point operation are composed in Figs. 5 and 6, separately. In each selection age of every subframe, the BAA sub formula in Fig. five are going to be dead 1st. each single remote consumer can criticism their channel additions to the baccalaureate in order that found the center price of square channel will increase may be computed as info contentions. Likewise, the amount of needed RBs for all the consumer sessions are going to be set to zero initially. when instatement, all the consumer sessions are going to be distributed one Rb to start with, to confirm least info rate conditions. Next, the remainder of the RBs are going to be assigned as indicated by the distribution metric. It plans to apportion the Rb to the consumer UN agency will best advantage in term of the vitality utilization diminish within the wake of obtaining the $\mathrm{Rb}$, and therefore the amount of needed RBs for the selected consumer are going to be enclosed one when the allotment. when the execution of BAA, the RBAA subalgorithm in Fig. half-dozen can during this manner be dead.

$$
\begin{gathered}
\operatorname{If}\left(\left(E_{k}>\gamma E_{\max }\right) \|\left(E_{k}<\rho E_{\max }\right)\right) \\
\operatorname{if}\left(\left(E_{k}>\gamma E_{\max }\right) \& \&(\text { level }<2)\right) \\
\text { level=level+1; } \\
\text { else if }\left(\left(E_{k}<\gamma E_{\max }\right) \& \&(\text { level }>0)\right) \\
\text { level=level-1; } \\
\text { end } \\
\text { end } \\
\text { NRT users }
\end{gathered}
$$

Set their capacities according to the level ; end

Fig. 3:- Pseudo code of EARCA

In RBAA, channel picks up and the quantity of each client session' required RBs are utilized as info contentions. For every RB, the subalgorithm means to discover the client who has the biggest channel pick up among all the clients. In the wake of finding the client, check whether the quantity of the current allotted RBs of the client equivalents to the quantity of its required RBs. In the event that yes, set the channel increase of the client approach to 0 , and discover another client whose channel increase is the biggest among every one of the clients till the while circle is over. Once the two sub algorithms are done in grouping, each client session's accessible RBs are resolved.

(F) Component Carrier Activation Algorithm:-

The CC initiation calculation is to make a decision the helpful utilization of the SCC as indicated by the unsteady system activity burden to essentially moderate the first vitality utilization of the SB. 
$/ \overline{\left|\boldsymbol{H}_{j \_}^{(\boldsymbol{k})}\right|}: 1$ the average squared channel gain across all $\mathrm{j}$ sub channels for user session $\mathrm{n}$ on $\mathrm{CC} \mathrm{k}$, which is expressed

$$
\overline{\mid \boldsymbol{H}_{\boldsymbol{j}_{n} \boldsymbol{n}}^{(\boldsymbol{k})}} \mid=\frac{1}{j} \sum_{j=1}^{j} \overline{\left|\boldsymbol{H}_{\boldsymbol{j}_{-} \boldsymbol{n}}^{(\boldsymbol{k})}\right|} /
$$

$\forall$ users $\in C C k$

Allocate each user session $1 \mathrm{RB}$;

While $\left(\sum_{n=1}^{N_{k}} \boldsymbol{m}_{\boldsymbol{n}}^{(\boldsymbol{k})}<\mathbf{2} \boldsymbol{j}\right)$

For $\mathrm{n}=1: N_{k}$

Calculate the allocation metric expressed as

$$
\begin{aligned}
& G_{n}^{(k)}=\frac{\beta N_{0}}{\boldsymbol{K}\left|\boldsymbol{H}_{j_{-}}^{(k)}\right|}\left[\left(\boldsymbol{m}_{n}^{(k)}+\mathbf{1}\right) \cdot \mathbf{2}^{\frac{r_{n}^{(k)}}{\beta\left(\left(m_{n}^{(k)}+1\right)\right.}}-\right. \\
& \left.\boldsymbol{m}_{n}^{(k)} \cdot \mathbf{2}^{\frac{r_{n}^{(k)}}{\beta\left(m_{n}^{(k)}\right)}}\right] ; \\
& \text { End }
\end{aligned}
$$

$$
\begin{aligned}
& n^{*}=\arg \min _{n} G_{n}^{(k)} ; \\
& m_{n *}^{(k)}=m_{n *}^{(k)}+1 ;
\end{aligned}
$$

end

Fig. 4:- Pseudo code of $B A A$

$/ S_{n}^{(k)}$ : the set of current allocated RBs for user session $\mathrm{n}$ on $\mathrm{CC} \mathrm{K} /$

For each $(m, j)_{R B}$

$$
n^{*}=\arg \max _{n}\left|H_{j_{-} n}^{(k)}\right|^{2} ;
$$

While $\left(\left|S_{n}^{(k)}\right|=m_{n^{*}}^{(k)}\right)$

$$
\begin{gathered}
\left|H_{j_{-} n}^{(k)}\right|^{2}=0 ; \\
n^{*}=\arg \max _{n}\left|H_{j_{-} n}^{(k)}\right|^{2} ;
\end{gathered}
$$

End

$$
S_{n^{*}}^{(k)}=S_{n^{*}}^{(k)} \cup\left\{(m, j)_{R B^{*}}\right\}
$$

End

Fig. 5:- Pseudo code of $R B A A$

\section{Results:-}

\section{A. Proposed method:-}

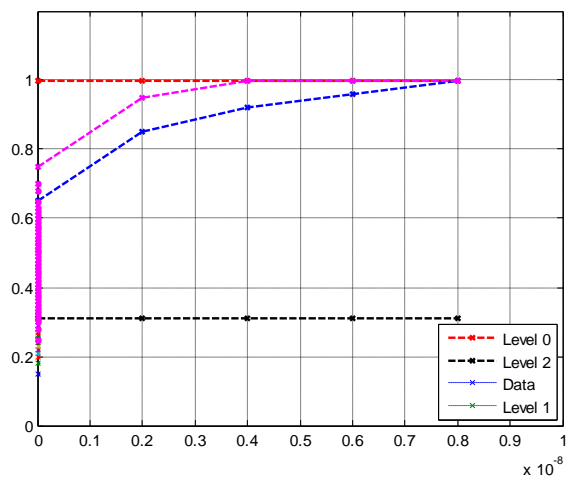

Figure 6:- Illustration of the reduction ratio as a function of the channel gain being used to determine the allocating capacity for the NRT users 


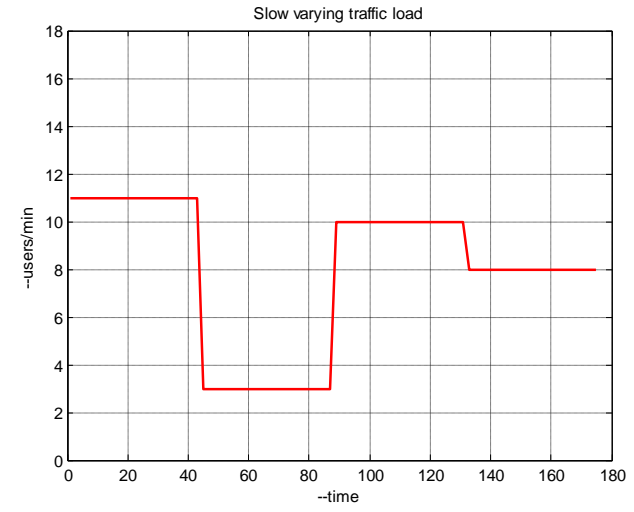

Figure 7:- Slow time-varying traffic loads versus time

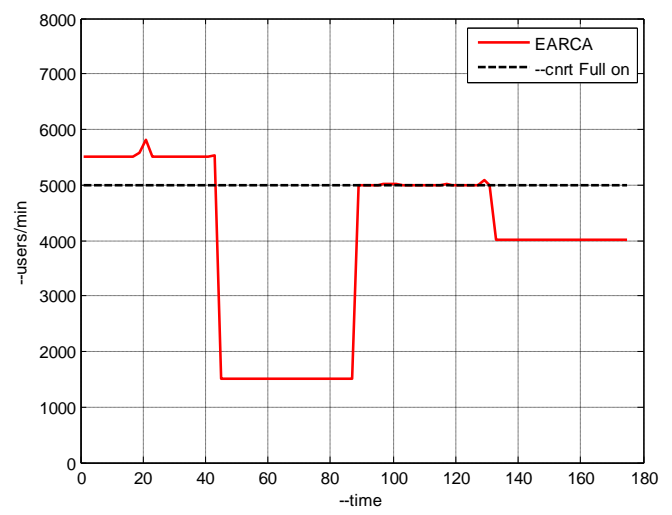

Figure 8:- Comparison of the energy consumption between the proposed scheme with EARCA, Level 2, and the comparison scheme.

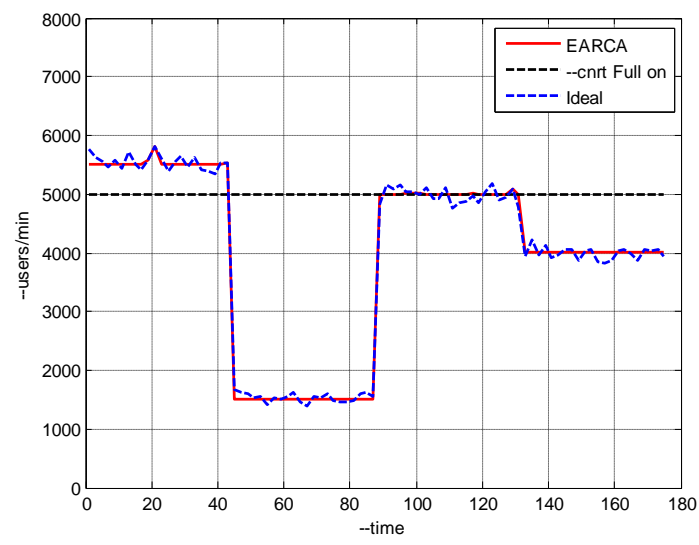

Figure 9:- Comparison of the energy consumption between the proposed scheme with EARCA, Level 0, and the comparison scheme. 


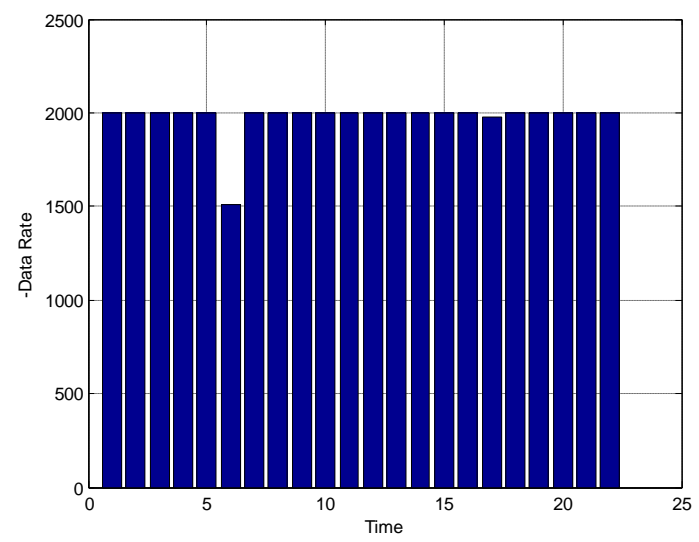

Figure 10:- NRT users' average data rate every 10 minutes of the proposed scheme with EARCA.

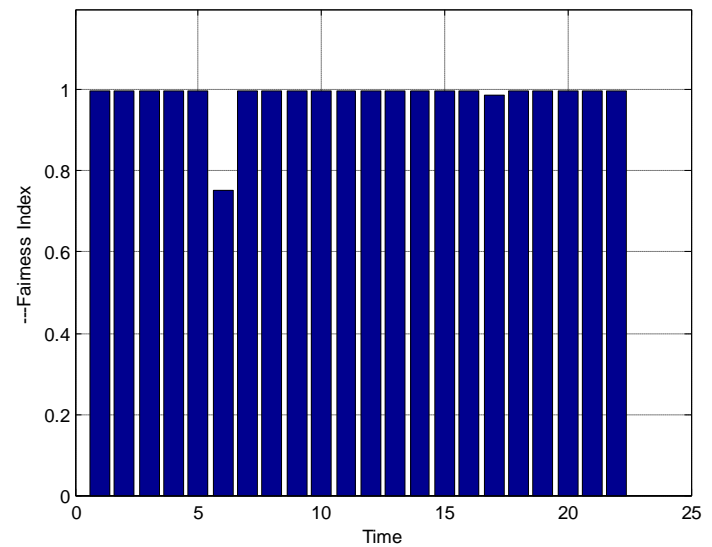

\section{B.SUI channel}

Figure 11:- Fairness index of the proposed scheme.

SUI channel is nothing but fixed path that is used for transmission with efficient manner.We are going to consider some parameters like Antenna diversity, Antenna correlation.

\section{Conclusion:-}

In this paper we have a tendency to develop an OFDMA based mostly multicarrier network systems with the assistance of SUI channel model, was with success projected. we have a tendency to are allocating resource blocks adaptively to manage the speed and power as smart QoS and also the fairness at constant time. By the employment of SCC, we have a tendency to avoiding needless energy consumption for the unsteady traffic load. With the assistance of simulations we have a tendency to ascertain that energy is expeditiously utilized giving glorious theme to use multi-cc cellular system at Bachelor of Science, mostly to avoid the issues like carbon di-oxide emission and increasing needs of the energy price. Also, with the assistance of SUI channel we have a tendency to are becoming higher enhanced results as compare to AWGN channel model. 


\section{References:-}

1. G. Yuan, X. Zhang, W. Wang, and Y. Yang, "Carrier aggregation for LTE-advanced mobile communication systems," IEEE Commun. Mag., vol. 48, no. 2, pp. 88-93, Feb. 2010.

2. Ghosh, R. Ratasuk, B. Mondal, N. Mangalvedhe, and T. Thomas, "LTE-advanced: Next-generation wireless broadband technology," IEEE Wireless Commun., vol. 17, no. 3, pp. 10-22, Jun. 2010.

3. L. M. Correia, D. Zeller, O. Blume, D. Ferling, Y. Jading, I. Go ' 1dor, G. Auer, and L. Van der Perre, "Challenges and enabling technologies for energy aware mobile radio networks," IEEE Commun. Mag., vol. 48, no. 11, pp. 66-72, Nov. 2010.

4. V. Mancuso and S. Alouf, "Reducing costs and pollution in cellular networks," IEEE Commun. Mag., vol. 49, no. 8, pp. 63-71, Aug. 2011.

5. J. Baliga, R. Ayre, K. Hinton, and R. S. Tucker, "Energy consumption in wired and wireless access networks," IEEE Commun. Mag., vol. 49, no. 6, pp. 70-77, Jun. 2011.

6. C. Y. Wong, R. S. Cheng, K. B. Lataief, and R. D. Murch, "Multiuser OFDM with adaptive subcarrier, bit, and power allocation," IEEE J. Select. Areas Commun., vol. 17, no. 10, pp. 1747-1758, Oct. 1999.

7. Z. Shen, J. G. Andrews, and B. L. Evans, "Optimal power allocation in multiuser OFDM systems," in Proc. IEEE GLOBECOM, San Francisco, CA, USA, Dec. 2003, pp. 337-341.

8. S. S. Jeong, D. G. Jeong, and W. S. Jeon, "Cross-layer design of packet scheduling and resource allocation in OFDMA wireless multimedia networks," in Proc. IEEE VTC Spring, Melbourne, Vic., May 2006, pp. 309-313. 